\title{
LINC01213 promotes prostate cancer cell progression through miR-597/ BCL2L1 axis
}

\author{
Xian Zhao \\ Taikang Tongji (Wuhan) Hospital \\ Xiaojing Xu \\ Wuhan First Hospital \\ Qiong Wang \\ Wuhan One Plus One Plastic Surgery Hospital \\ Xiaofei Wu ( $\nabla$ xiao0fei7@163.com ) \\ Taikang Tongji (Wuhan) Hospital
}

\section{Research Article}

Keywords: LINC01213, prostate cancer. miR-597, BCL2L1

Posted Date: March 16th, 2021

DOI: https://doi.org/10.21203/rs.3.rs-289818/v1

License: (9) This work is licensed under a Creative Commons Attribution 4.0 International License. Read Full License 


\section{Abstract}

Background: Majority of cancer related deaths in males are attributed to prostate cancer (PRAD) throughout the world. Recently, the role of long non-coding RNAs (IncRNAs) in the pathogenesis of cancer has been widely explored. In this study, we investigated the role of IncRNA LINC01213 (LINC01213) in tumorigenesis of prostate cancer (PRAD).

Methods: PRAD and adjacent tissue samples were collected from cancer patients. Survival rate among these patients was compared by Kaplan-Meier analysis. PRAD cells viability was estimated by CCK-8 method while AnnexinV/PI cytometry assay was used to determine the percent of apoptotic cells. qRTPCR and western blot assay were used to determine the mRNA and protein expressions, respectively. Interaction between LINC01213 and corresponding miRNA as well as between miRNA and mRNA was confirmed by dual luciferase reporter gene assay. PRAD cells were also injected subcutaneously in nude mice to support in vitro findings.

Results: It was observed that LINC01213 was highly expressed in PRAD samples and cell lines. Downregulation of LINC01213 in PRAD cells decreased cell viability and inhibited proliferation. Luciferase reporter gene assay and RNA pull-down confirmed that LINC01213 targeted miR-597-3p. Increased expression of miR-597-3p resulted in decreased BCL2L2 expression in vitro. Inhibitory effects of miR-597$3 p$ on PRAD cells' survival and growth were diminished after LINC01213 overexpression which was also associated with alteration in the protein expression of BCL-xL, BCL-2 as well as caspase 3 and caspase 9.

Conclusion: Taken together, our findings suggest that LINC01213 plays its role in PRAD tumorigenesis through miR-597-3p/ BCL2L2 dependent pathway with associated modulation of genes involved in cell survival and apoptosis.

\section{Introduction}

Prostate cancer (PRAD) is one of the leading causes of cancer related deaths throughout the world and the most diagnosed type of cancer in men [1]. It has been shown that almost 1.6 million people are diagnosed with PRAD each year [2]. The incidence of PRAD has shown interesting differences across the globe. For instance, African-Americans' have the highest incidence of PRAD while the lowest incidence has reported in Asian men [1,3]. Moreover, the odds of PRAD diagnosis in less developed countries are 1 in 47 than 1 in 6 in developed ones [4]. This discrepancy may be explained by early detection of prostatespecific antigen (PSA) in develop countries and the differences in lifestyle. Early detection of PRAD might help in the removal of the localized tumor through radiation and surgical removal but may inversely effect the quality of life [5].

The abnormal expression of IncRNA is closely related to the occurrence and development of tumors. LncRNAs adsorb miRNA through sponging and regulate the expression of downstream genes, thereby, play important roles in the occurrence and development of tumors [6]. For instance, MEG3 has been shown to act as tumor suppressor at human pituitary tumor derived cell line [7]. LncRNA ADAMTS9-AS2 
has been shown to inhibit glioma cell migration [8]. LncRNA PVT1 have been shown to regulate the growth and predictor of prognosis in prostate cancer [9]. IncRNA UCA1 has been shown to promote to proliferation and tumor metastasis in gastric cancer [10]. LncRNA SNHG16 has been shown to promote tumor growth of pancreatic cancer [11]. LncRNA H19 has been shown to promote tumor progression in cervical cancer by targeting miR-138-5p [12].

It has been reported that LINC01213 plays a role in promoting melanoma and breast cancer $[13,14]$. However, no report is available that investigates the role of LINC01213 in prostate cancer. According to the analysis of TCGA database, the expression of LINC01213 in cancer tissues is generally higher than that in normal tissues. IncBASE. 2 predicts that LINC01213 can target miR-597-3p, and miR-597 has been shown to be involved in a variety of cancer types such as breast cancer [15] and colon cancer [16]. miRDB predicts that BCL2L1 is the target gene of mir-597-3p, and BCL2L1(BCL-XL) is a member of BCl2 family, which participates in the regulation of apoptosis. We took the high expression of LINC01213 in prostate cancer as the breakthrough point, and explored by silencing LINC01213 in prostate cancer cell line.

\section{Methods}

\section{Cell culture}

RWPE-1 cells were grown in Keratinocyte Serum Free Medium (Gibco, Invitrogen, USA) supplemented with bovine pituitary extract and human recombinant epidermal growth factor to make the complete medium. C4-2 cells were grown in Dulbecco's Modified Eagle' medium (DMEM): F12 medium (1:1) (Invitrogen, USA), LNCaP and 22Rv1cells were maintained in RPMI1640 (Invitrogen, USA) while DU 145 cells were maintained in Eagle's Minimum Essential Medium (Thermo Fisher Scientific, USA), and PC-3 cells were grown in F-12K medium (Thermo Fisher Scientific, USA). Except for RWPE-1 cells, all other mediums were supplemented with $10 \%$ fetal bovine serum and $1 \%$ penicillin-streptomycin (Gibco- Thermo Fisher Scientific, USA). All the cells were cultured under standard laboratory conditions in an incubator at $37^{\circ} \mathrm{C}$ with $5 \% \mathrm{CO}_{2}$ supply.

\section{Collection of clinical samples}

60 pairs of tissue samples from PRAD patients were collected at Taikang Tongji (Wuhan) Hospital. Samples were collected after surgical removal of PRAD and adjacent tissue samples which were immediately stored at $-80^{\circ} \mathrm{C}$ until further use. All the clinical procedures were in accordance with the declaration of Helsinki and written informed consents were collected from all the participants of the study. All the protocols were reviewed and approved by the committee for human experimentations, Taikang Tongji (Wuhan) Hospital, China.

\section{Cell viability assay}

Cell viability was determined by the cell counting kit 8 assay (CCK-8). Briefly, $6 \times 10^{3}$ cells were seeded into 96 well cell culture plates and cultured for an overnight. Different treatments were applied and cells 
were incubated for 24,48 , and $72 \mathrm{~h}$. At the end of incubation period, $10 \mu \mathrm{L}$ of CCK-8 (Dojindo, Japan) was added to each well and further for $1 \mathrm{~h}$ at $37^{\circ} \mathrm{C}$. Finally, the absorbance was measured at $450 \mathrm{~nm}$ wavelength using Elx800 absorbance reader (BioTek Instruments).

\section{Colony formation assay}

This assay was conducted to investigate the proliferation capacity of the cells. For this purpose, PRAD cells transfected or not were seeded onto 24-well plates with 500 cells per well. Cells were cultured for 10 days under standard laboratory conditions. The, cell were fixed with $100 \%$ methanol (Sigma, USA) and colonies were visualized under laboratory microscope (Olympus, Japan)after staining with $0.1 \%$ crystal violet solution (Solarbio, China).

\section{Western blot assay}

Collected cells were lysed in ice cold RIPA buffer (Servicebio, China) with added protease inhibitor cocktail (Sigma, USA). Protein content of samples was determined by using BCA assay kit and proteins were separated by electrophoresis in 4\%-20\% polyacrylamide gels (GenScript). Afterwards, the proteins were transferred to polyvinylidene fluoride membranes (Millipore, USA) followed by saturation in $5 \%$ skim milk at room temperature. Then, primary antibody (1:500) was added to the membranes and incubated for an overnight at $4^{\circ} \mathrm{C}$. Then, the secondary antibodies (1:2000) and incubated at room temperature for $2 \mathrm{~h}$. Protein blots were detected using Enhanced Chemiluminescence Kit (FDbio Science, China).

\section{Flow cytometery assay}

Cells were collected after $72 \mathrm{~h}$ of transfection or not and washed three times with PBS. Cells were then incubated in dark with FITC-Annexin V and propidium iodide (Liankebio, China) for 15 min. Flow

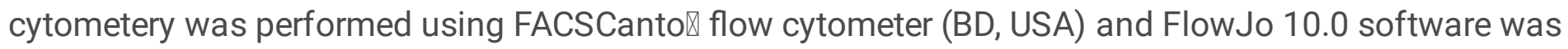
used to determine the percent of apoptotic cells.

\section{Subcutaneous tumorigenesis assay in nude mice}

BALB/c male nude mice weighing approximately 22-25 g were purchased form the Zhejiang Chinese Medical University (Hangzhou, China). Mice were acclimatized for at least a week under standard laboratory conditions. Afterwards, $2 \times 10^{6}$ PRAD cells suspended in 200 cells $\mu \mathrm{L}$ of PBS were subcutaneously injected into the left flank of the mice. Tumor xenograft diameter was measured after each week for a total of 4 weeks for determining the tumor volume using $V=\pi / 6 \times$ length $\times$ width $^{2}$ and tumor weight was also determined at the end of this period. All the experimental protocols were approved by the animal ethical committee of Taikang Tongji (Wuhan) Hospital and were in compliance with the ARRIVE guidelines.

\section{Quantitative reverse transcription polymerase chain reaction (RT-qPCR)}


TRIzol reagent (Invitrogen, USA) was used for the extraction of total RNA from the samples and the RNA concentrations were determined by using nano-drop spectrophotometer. High-Capacity cDNA Reverse Transcription Kit (Thermo Fisher Scientific, USA) was used to reverse transcribe RNA to cDNA. This was followed by PCR for 40 cycles of alternate temperatures of denaturation, annealing and extension. Gene expression was analyzed employing SYBR Green PCR master mix (Thermo Fisher Scientific, USA) using $2^{-\Delta \Delta C t}$ method. Product specificity was analyzed by melt-curve analysis and all the experiments were replicated at-least in triplicate.

\section{Luciferase reporter gene assay}

Approximately $5 \times 10^{4}$ cells were seeded onto a 96 -well plate for $24 \mathrm{~h}$ followed by transfection/cotransfection for $48 \mathrm{~h}$ with Lipofectamine 2000 (Invitrogen, USA) according to the manufacturer's guidelines. Luciferase assay kit (Promega, USA) was used to perform the luciferase activity according to the provided protocols. In brief, to a volume of $100 \mu \mathrm{L}$ containing cells an equal volume of luciferase assay solution was added and incubated for $20 \mathrm{~min}$ at room temperature. Microplate reader (Synergy H4 Hybrid Reader, BioTek, Winooski, USA) was used to measure luciferase activities [17].

\section{RNA pull-down assay}

Previously described procedures were used for the RNA pull-down assay [18]. This assay was conducted according to the previously described procedures. Biotin-labeled bio- LINC01213 probe was provided by Sangon Biotech (Shanghai, China). Cells were collected and lysed. One part of the lysate was kept to be used for input control. The other part was incubated with magnetic Dynabeads M-280 Streptavidin beads (Invitrogen, Carlsbad, CA, USA) beads for an overnight at $4{ }^{\circ} \mathrm{C}$. qRT-PCR assay was used to determine the miR-597-3p enrichment.

\section{Statistical analysis}

Statistical analysis of the results was conducted by the GraphPad prism software version 6. KaplanMeier analysis was used to compare the survival difference between patient groups. Student's $t$ test was used for two groups while One-way ANOVA was used for the statistical analysis of more than two groups. $P$ values less than 0.05 were considered significant. The data represent the mean \pm SD of at-least three independent experiments.

\section{Results}

\section{LINC01213 is highly expressed in PRAD tissues and cell lines}

TGCA database was used to analyze the expression level of LINC01213 in PRAD tissue samples. It was observed that LINC01213 had significantly increased $(P<0.05)$ expression in PRAD tissue than the normal controls (Fig.1A). To further confirm these findings, 66 pairs of PRAD clinical samples with normal adjacent tissues were collected. qRT-PCR analysis showed that PRAD tissue samples exhibited 
significantly increased $(\mathrm{P}<0.01)$ expression of LINC01213 (Fig.1B). The median expression value of LINC01213 in PRAD tissue in Fig.1B was taken as the cut-off value. On the basis of this value 66 PRAD patients were divided into high and low expression groups of LINC01213, each containing 33 patients. Kaplan-Meier survival curve was used to evaluate the overall survival rate of these two groups. It was observed that high expression of LINC01213 was associated with significantly less $(P=0.0345)$ survival than the low expression (Fig.1C). Furthermore, the expression level of LINC01213 in PRAD cell lines LNCaP, 22Rv1, C4-2, DU145, PC-3 and normal prostate epithelial cell line RWPE-1 was detected by qRTPCR. It was observed that tumor cell lines exhibited significantly increased $(P<0.01)$ expression of LINC01213 than the normal cells (Fig.1D). The relationship between LINC01213 expression and clinical pathological data was analyzed by Chi-square test (Table1). Data suggested that LINC01213 expression was closely related to clinical stage, lymph node metastasis and tumor size $(P<0.05)$ but was independent of the patient's age and gender.

\section{Silencing LINC01213 inhibited the proliferation of PRAD cells and promoted the apoptosis of PRAD cells}

Two cell lines with the highest expression of LINC01213 in Fig.1D i.e. LNCaP and PC-3 were selected to establish a stable LINC01213 knock-down and the silencing efficiency $>50 \%$ was confirmed by qRT-PCR (Fig.2A). It was observed that LINC01213 knock-down resulted in significantly decreased $(P<0.01)$ survival of LNCaP and PC-3 as indicated by OD values during CCK-8 assay (Fig.2B). In addition, LINC01213 knock-down significantly reduced the proliferation ability of LNCaP and PC-3 cells during colony formation assay (Fig.2C) and increased the rate of apoptosis (Fig.2D). Interesting, LINC01213 resulted in significantly reduced $(P<0.01)$ subcutaneous tumor growth in nude mice both in terms of volume (Fig.2E) and weight (Fig.2F) during 28 days of observation.

\section{LINC001213 targets miR-597-3p}

Web based tool LNCbase V2 online database predicted the presence of miR-597-3p binding sites in LINC01213 (Fig.3A). Luciferase experiment proved that LINC01213 interacted with miR-597-3p where it was observed that compared with miR-NC, overexpression of miR-597-3p could inhibit the luciferase intensity of wild-type LINC001213 vector which was restored after the mutation of the predicted binding site (Fig.3B). In addition, RNA pull- down test showed that LINC01213 probe pulled-down significantly more miR-597-3p, compared with oligo probe (Fig.3C). Furthermore, LINC01213 knock-down resulted in significantly increased $(P<0.01)$ expression of miR-597-3p than the control (Fig.3D). Interestingly, PRAD tissues expressed significantly decreased $(P<0.01)$ expression of miR-597-3p (Fig.3E) and an inverse correlation $\left(R^{2}=0.4165, P<0.01\right)$ was observed between miR-597-3p and LINC01213 in these samples (Fig.3F).

\section{MiR-597-3p targeted BCL2L1}

Online database miRDB predicted the presence of miR-597-3P binding sites in BCL2L1 3'UTR (Fig.4A). To further confirm these findings, luciferase reporter gene experiment was performed. It was observed that overexpression of miR-597-3P could inhibit the luciferase intensity of wild-type BCL2L1 vector. In 
contrast, the inhibitory effect disappeared after the mutation of the predicted binding site of BCL2L1 (Fig.4B). In addition, miR-597-3p mimics significantly decreased $(P<0.01)$ the expression of BCL2L1 as indicated by qRT-PCR (Fig.4C) and western blot (Fig.4D) in PC-3 and LNCaP cells. Interestingly, PRAD tissue samples exhibited significantly increased $(P<0.01)$ expression of BCL2L1 than the adjacent tissues (Fig.4E) and an inverse correlation $\left(R^{2}=0.6616, P<0.01\right)$ was observed between miR-597-3p and BCL2L1 expression (Fig.4F).

\section{LINC01213 promotes PRAD through miR-597-3P/BCL2L1 axis}

To further unveil the mechanism of LINC01213, pcDNA3.1-LINC01213 was used to overexpress the LINC01213 and the efficacy was confirmed by qRT-PCR (Fig.5A). Henceforth, the expression level of BCL2L1 was detected by qRT-PCR in different groups of LNCaP and PC-3 cells (miR-NC, miR-597-3p mimics, miR-597-3p mimics+pcDNA3.1-LINC01213). The results showed that overexpression of miR-597$3 p$ decreased the expression level of BCL2L1, while co-transfection pcDNA3.1-LINC01213 restored the expression of BCL2L1 in these cells (Fig.5B). Changes in cell viability were determined by CCK-8 assay which showed that overexpression of miR-597-3p reduced PRAD cell viability and this effect was curtailed after LINC01213 overexpression in these cells (Fig.5C). Similarly, miR-597-3p significantly $(P<$ 0.001 ) reduced the colony formation (Fig.5D) and increased the rate of apoptosis (Fig.5E) of LNCaP and PC-3 cells while LINC01213 overexpression reversed these effects. Henceforth, western blot method was employed to investigate the effect of miR-597-3p overexpression on the expression of proteins involved in cell proliferation and apoptosis. It was observed that miR-597-3p overexpression reduced the protein expression of BCL-xL and BCL-2 while the protein expression of cleaved-caspase 3 and cleavedCASPASE9 were significantly increased in LNCaP and PC-3 cells (Fig.5F). In contrast, the protein expression of caspase3, caspase9 as well as GAPDH remained unaffected (Fig.5F).

\section{Discussion}

It was observed that LINC01213 played a crucial in the pathogenesis of PRAD as it was highly expressed on the PRAD clinical samples as well as on cell lines. We used mechanistic approach in this study which revealed that LINC01213 could exert its role on PRAD cells through miR-597-3p and BCL2L1 dependent pathway. Tumor inhibiting properties of miR-597-3p have been previously reported by Wen et al. who have shown that this miRNA could inhibit the proliferation and invasion of thyroid carcinoma SW579 cells by modulating the expression of RAB23 [19]. In the current report, miR-597-3p overexpression adversely a affected the survival and proliferation of PRAD cell lines which is in corroboration of the report by Wen et al.

Our results indicate that LINC01213 targeted BCL2L1 protein and also modulated the expression of other proteins involved in cell survival and apoptosis. BCL2L1 gene has been previously implicated in the pathogenesis of various cancers [20,21]. For instance, Sillars-Hardebol et al. have reported that BCL2L1 played a functional role in colorectal cancer [22]. Yang et al. have shown that IncRNA LINC02595 promoted colorectal cancer progression through the inhibition of miR-203b-3p and thereby upregulating 
BCL2L1 expression [23]. Another study by Ostadrahimi et al. has shown that BCL2L1 gene was upregulated in PRAD tissue samples and cell lines which is also in corroboration of the current findings [24]. BCL2 and BCL-xL are the members of BCL-2 family of proteins that are known to exert anti-apoptotic effects and promote cell survival [25]. In contrast, Caspase 3 and caspase 9 belong to the caspase family of cysteine proteases that have been implicated in apoptosis. Brentnall et al. have previously reported that caspase 3 and caspase 9 play distinct roles during intrinsic apoptosis. Hence, caspase 9 can be involved in initiation of apoptosis while caspase 3 plays a role in execution [26]. It was observed that miR597-3p overexpression in PRAD cell lines decreased the protein expression of anti-apoptotic factors (BCL2 and BCL-xL) and increased the expression of pro-apoptotic agents (caspase 3 and caspase 9), thereby, promoting apoptosis that was manifested by reduced survival and proliferation of PRAD cells lines. In contrast, LINC01213 overexpression in these cells reversed the effects of miR-597-3p overexpression in these cells by increasing the expression of anti-apoptotic factors. A previous study by Zhang et al. has shown that miR-608 suppressed the progression of prostate cancer by targeting the BCL2L1/caspase-3 pathway. Another report by Rachel et al. has suggested that caspase-3 protein expression was lost in human prostate cancer which served to promote the prostate tumorigenesis [27]. Rodríguez-Berriguete et al. have shown that immunoreactivity to caspase-3 and caspase-9 is frequently lost in human prostate tumors which further strengthen our findings [28]. Taken together, our findings suggest that LINC01213 modulates the progression of PRAD through the modulation of miR-597-3p/ BCL2L1 dependent pathway. Furthermore, LINC01213 may serve as the biomarker of PRAD and also a prognostic indicator of the disease. We propose LINC01213 as a novel target for future RNA based therapeutic approaches for the treatment of PRAD.

\section{Declarations}

\section{Ethics approval}

60 pairs of tissue samples from PRAD patients were collected at Taikang Tongji (Wuhan) Hospital. Samples were collected after surgical removal of PRAD and adjacent tissue samples which were immediately stored at $-80^{\circ} \mathrm{C}$ until further use. All the clinical procedures were in accordance with the declaration of Helsinki

\section{Consent to participate}

The written INFORMED CONSENTS were collected from all the participants of the study. All the protocols were reviewed and approved by the committee for human experimentations, Taikang Tongji (Wuhan) Hospital, China.

\section{Consent for publication}

All authors consent to publication.

\section{Availability of data and materials}


All supporting data of this work, which are not available in public because of the ethical restrictions are available from the corresponding author upon request.

\section{Competing interests}

The authors report no conflicts of interest in this work.

\section{Funding}

There is no funding source in this work.

\section{Authors' contributions}

Xian Zhao designed the project and collected data. Xiaojing Xu analyzed the data and drafted the manuscript. Qiong Wang did almost all the experiments and were involved in data collection and analysis. Xiaofei Wu conducted methodology and validation and was responsible for investigation and data curation. All the authors revised and corrected the manuscript.

\section{Acknowledgements}

We thank the reviewers for their constructive comments.

\section{References}

1. Pernar $\mathrm{CH}$, Ebot EM, Wilson KM, et al. The epidemiology of prostate cancer. Cold Spring Harb Perspect Med 2018;8:a030361.

2. Fitzmaurice $\mathrm{C}$, Allen C, Barber RM, et al. Global, regional, and national cancer incidence, mortality, years of life lost, years lived with disability, and disability-adjusted life-years for 32 cancer groups, 1990 to 2015: a systematic analysis for the global burden of disease study. JAMA Oncol 2017;3:524-48.

3. Ferlay J, Soerjomataram I, Ervik M. Cancer incidence and mortality worldwide, international agency for research on cancer. Cancer Incid Mortal worldwide, Int agency Res cancer 2013;:120-63.

4. Chu KC, Tarone RE, Freeman HP. Trends in prostate cancer mortality among black men and white men in the United States. Cancer Interdiscip Int J Am Cancer Soc 2003;97:1507-16.

5. Litwin MS, Tan H-J. The diagnosis and treatment of prostate cancer: a review. Jama 2017;317:2532-42.

6. Szilágyi M, Pös O, Márton É, et al. Circulating Cell-Free Nucleic Acids: Main Characteristics and Clinical Application. Int J Mol Sci 2020;21:6827.

7. Chunharojrith $P$, Nakayama $Y$, Jiang $X$, et al. Tumor suppression by MEG3 IncRNA in a human pituitary tumor derived cell line. Mol Cell Endocrinol 2015;416:27-35.

8. Yao J, Zhou B, Zhang J, et al. A new tumor suppressor LncRNA ADAMTS9-AS2 is regulated by DNMT1 and inhibits migration of glioma cells. Tumor Bio/2014;35:7935-44. 
9. Yang J, Li C, Mudd A, et al. LncRNA PVT1 predicts prognosis and regulates tumor growth in prostate cancer. Biosci Biotechnol Biochem 2017;81:2301-6.

10. Wang C-J, Zhu C-C, Xu J, et al. The IncRNA UCA1 promotes proliferation, migration, immune escape and inhibits apoptosis in gastric cancer by sponging anti-tumor miRNAs. Mol Cancer 2019;18:115.

11. Liu S, Zhang W, Liu K, et al. LncRNA SNHG16 promotes tumor growth of pancreatic cancer by targeting miR-218-5p. Biomed Pharmacother 2019;114:108862.

12. Ou L, Wang D, Zhang H, et al. Decreased expression of miR-138-5p by IncRNA H19 in cervical cancer promotes tumor proliferation. Oncol Res Featur Preclin Clin Cancer Ther 2018;26:401-10.

13. Yan H, Tan D, Xie P, et al. Multiple IncRNAs affect the incidence and development of melanoma. Zhong nan da xue xue bao Yi xue ban = J Cent South Univ Med Sci 2017;42:134-8.

14. Porsch $\mathrm{M}$, Özdemir $\mathrm{E}$, Wisniewski $\mathrm{M}$, et al. Time resolved gene expression analysis during tamoxifen adaption of MCF-7 cells identifies long non-coding RNAs with prognostic impact. RNA Biol 2019;16:661-74.

15. He J, Mai J, Li Y, et al. miR-597 inhibits breast cancer cell proliferation, migration and invasion through FOSL2. Oncol Rep 2017;37:2672-8.

16. Fei B, Li S, Zhuo Liu ZL, et al. MicroRNA (miR)-597-5p inhibits colon cancer cell migration and invasion by targeting FOS-Like antigen 2 (FOSL2). Front Oncol 2019;9:495.

17. Wan Y, Yang Z-Q. LncRNA NEAT1 affects inflammatory response by targeting miR-129-5p and regulating Notch signaling pathway in epilepsy. Cell Cycle 2020;19:419-31.

18. Cui J, Li W, Liu G, et al. A novel circular RNA, hsa_circ_0043278, acts as a potential biomarker and promotes non-small cell lung cancer cell proliferation and migration by regulating miR-520f. Artif cells, nanomedicine, Biotechnol 2019;47:810-21.

19. Wen HL, Xu ZM, Lin SY, et al. miR-597-3p inhibits invasion and migration of thyroid carcinoma SW579 cell by targeting RAB23. Endokrynol Pol 2020.

20. Obasi TC, Braicu C, lacob BC, et al. Securidaca-saponins are natural inhibitors of AKT, MCL-1, and BCL2L1 in cervical cancer cells. Cancer Manag Res 2018;10:5709.

21. Zhang $\mathrm{H}$, Xue J, Hessler $\mathrm{P}$, et al. Genomic analysis and selective small molecule inhibition identifies BCL-X L as a critical survival factor in a subset of colorectal cancer. Mol Cancer 2015;14:1-9.

22. Sillars-Hardebol AH, Carvalho B, Beliën JAM, et al. BCL2L1 has a functional role in colorectal cancer and its protein expression is associated with chromosome 20q gain. J Pathol 2012;226:442-50.

23. Yang Z, An Y, Wang N, et al. LINC02595 promotes tumor progression in colorectal cancer by inhibiting miR-203b-3p activity and facilitating BCL2L1 expression. J Cell Physiol 2020.

24. Ostadrahimi S, Fayaz S, Parvizhamidi $M$, et al. Downregulation of miR-1266-5P, miR-185-5P and miR-30c-2 in prostatic cancer tissue and cell lines. Oncol Lett 2018;15:8157-64.

25. Opferman JT, Kothari A. Anti-apoptotic BCL-2 family members in development. Cell Death Differ 2018;25:37-45. 
26. Brentnall M, Rodriguez-Menocal L, De Guevara RL, et al. Caspase-9, caspase-3 and caspase- 7 have distinct roles during intrinsic apoptosis. BMC Cell Biol 2013;14:32.

27. Winter RN, Kramer A, Borkowski A, et al. Loss of caspase-1 and caspase-3 protein expression in human prostate cancer. Cancer Res 2001;61:1227-32.

28. Rodríguez-Berriguete G, Galvis L, Fraile B, et al. Immunoreactivity to caspase-3, caspase-7, caspase-8, and caspase-9 forms is frequently lost in human prostate tumors. Hum Pathol 2012;43:229-37.

29. Legends

\section{Table}

Table 1: The relationship between LINC01213 expression and clinical pathological data

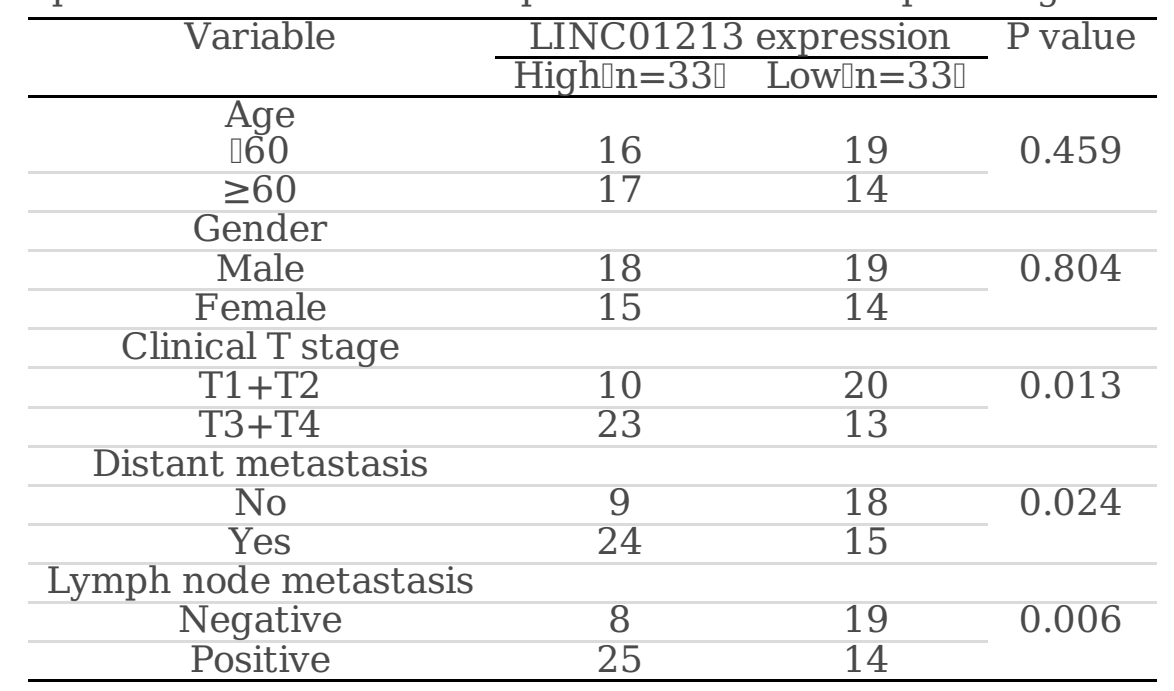

\section{Figures}

fig1

A

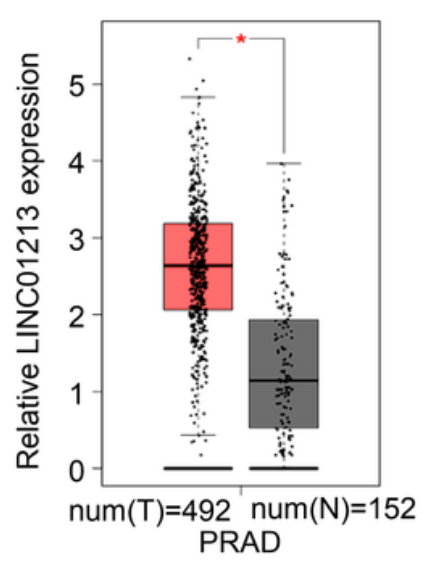

B

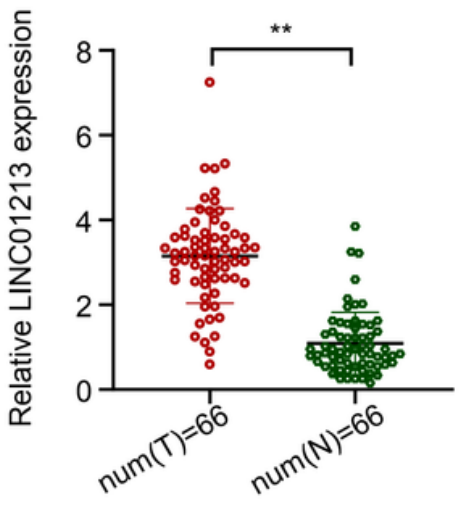

C

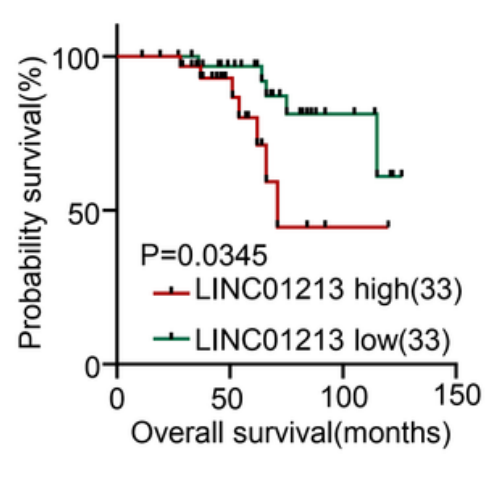

D

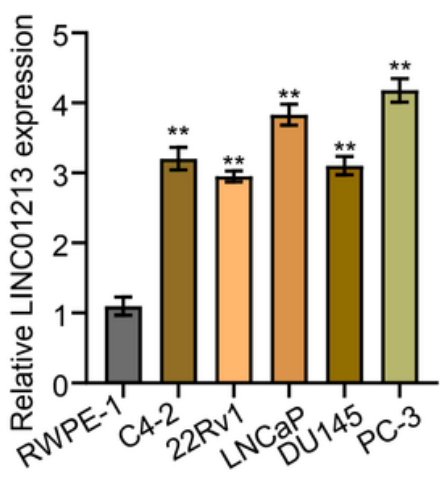

Figure 1 
Expression of LINC01213 in PRAD tissues and cell lines. (A) Expression level of LINC01213 in PRAD tissue samples according to TGCA database. (B) The expression level of LINC01213 in 66 pairs of PRAD clinical samples and adjacent tissues. (C) Kaplan-Meier survival curve comparing high and low expression groups of LINC01213. (D) The expression level of LINC01213 in PRAD cell lines (LNCaP, 22Rv1, C4-2, DU145, PC-3) and normal prostate epithelial cell line (RWPE-1). Statistical analysis has been described under the Materials and Methods section. ${ }^{*}=\mathrm{P}<0.05,{ }^{*}=\mathrm{P}<0.01$

fig2

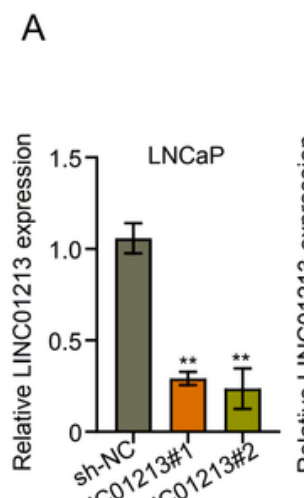

B
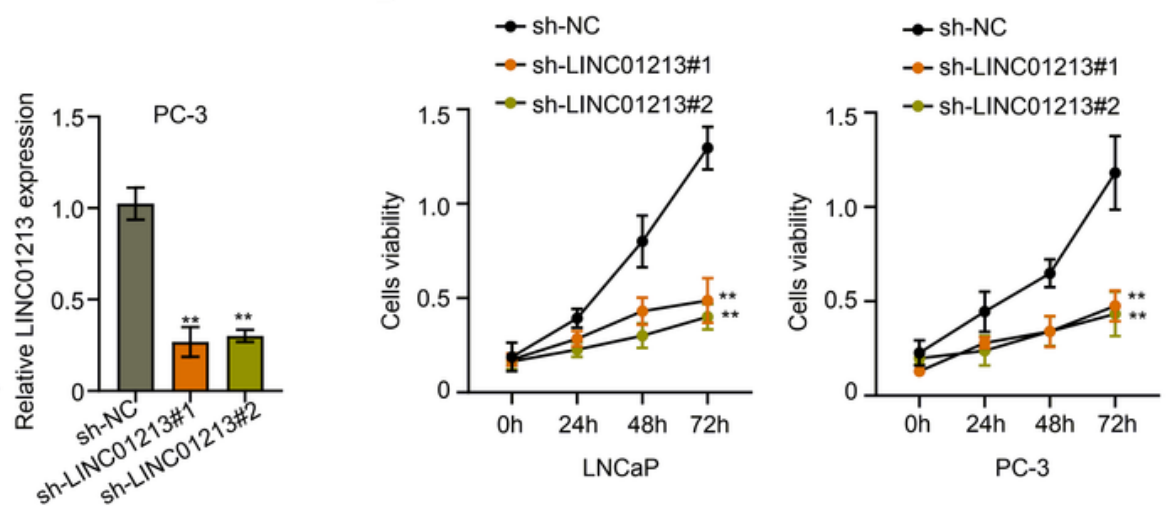

C
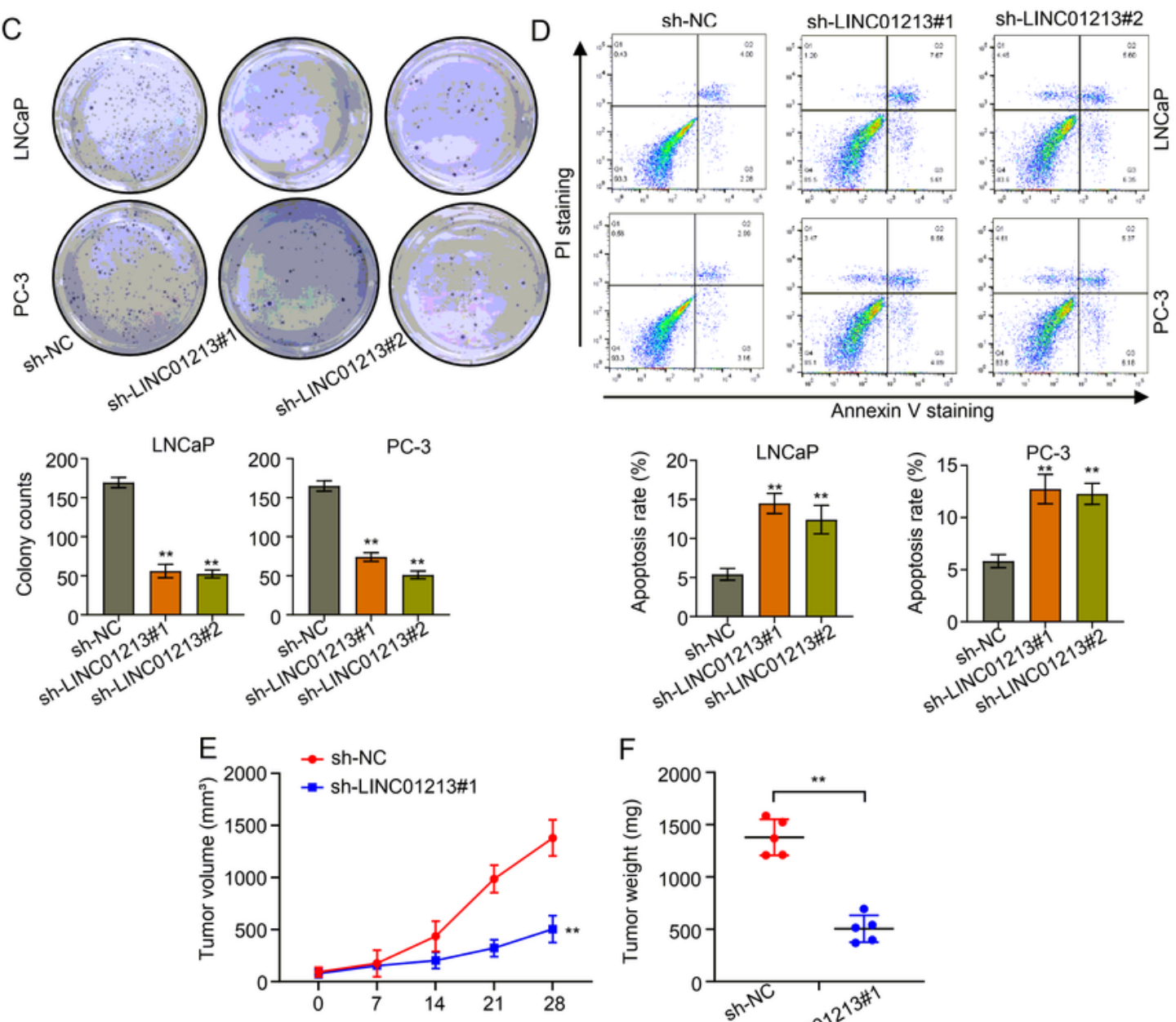

$\mathrm{F}$

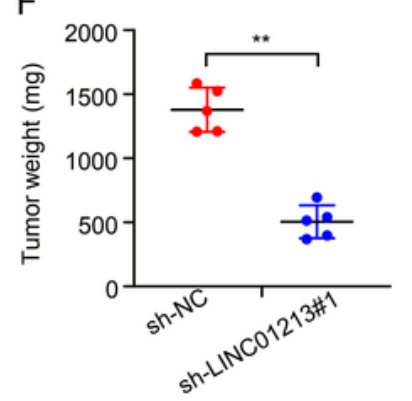

Figure 2 
Silencing LINC01213 inhibited the proliferation of PRAD cells and promoted cell apoptosis. (A) Two cell lines with the highest expression of LINC01213 (assuming LNCaP and PC-3) were selected and a stable knock-down LINC01213 cell line was established. (B) The viability of LNCaP and PC-3 cells in different groups was detected by CCK-8 assay. (C) Colony formation test of cell proliferation. (D) Annexin V/PI flow cytometery analysis to estimate the \% of apoptotic cells following different treatments. (E, F)

Subcutaneous tumor growth in nude mice expressed in terms of volume and weight during 28 days of observation. Statistical analysis has been described under the Materials and Methods section. $*=P<$ $0.05, * *=\mathrm{P}<0.01$

fig3

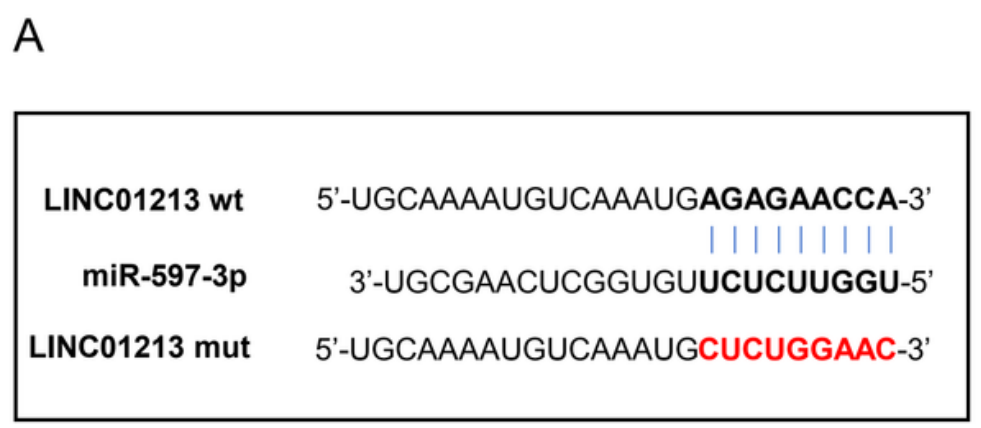

C

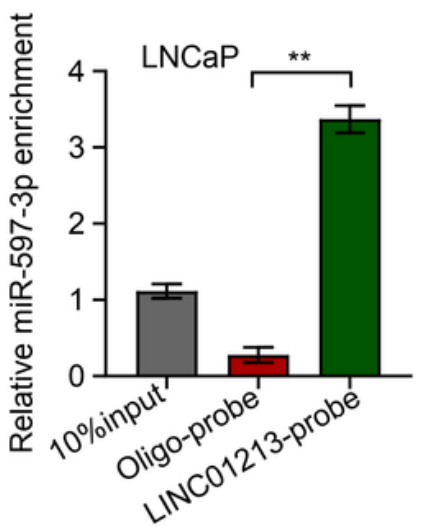

E

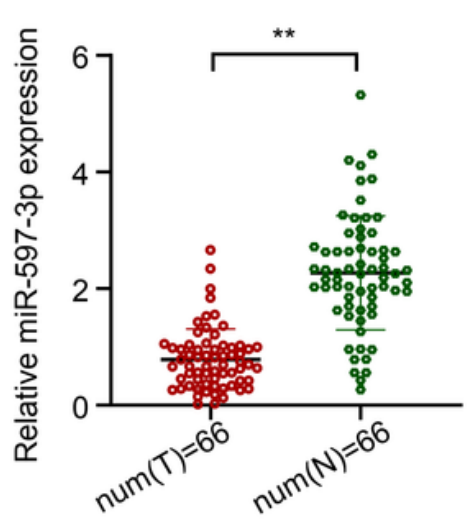

D

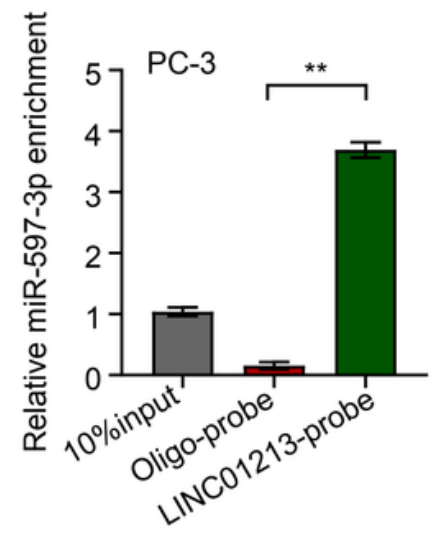

$\mathrm{F}$
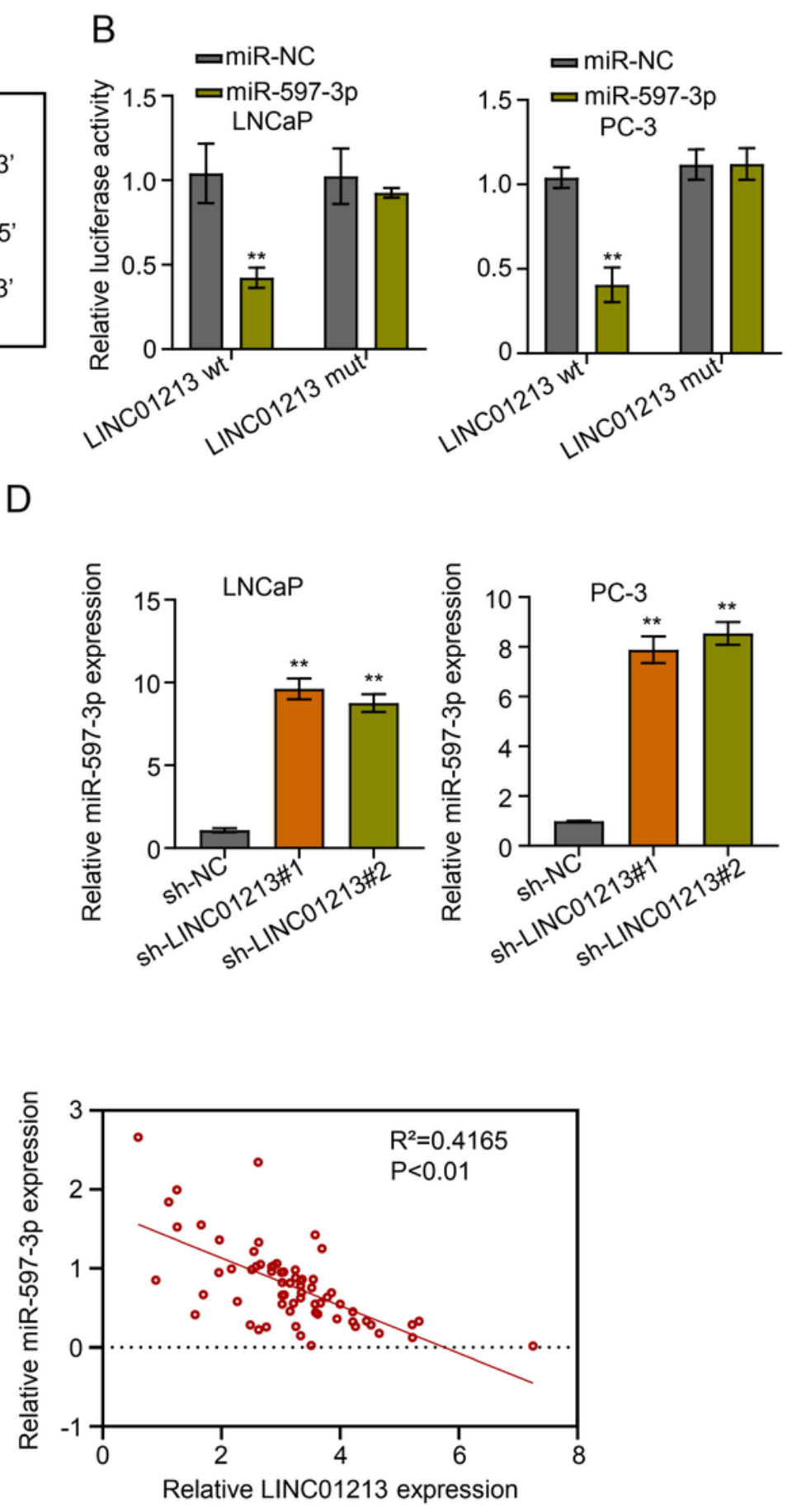
Figure 3

miR-597-3p is the target of LINC001213. (A) Web based tool LNCbaseV.2 indicated that LINC01213 had miR-597-3p binding sites. (B) Luciferase experiment to confirm that LINC01213 interacted with miR-5973p. (C) RNA pull-down assay. (D) Effect of sh-LINC01213 \# 1 and sh-linc01213 \# 2 medicated LINC01213 knock-down on miR-597-3p expression. (E) The expression level of miR-597-3p in 66 pairs of PRAD and normal tissues detected by qRT-PCR. (F) The expression of LINC01213 was negatively correlated with miR-597-3p in PRAD clinical samples. Statistical analysis has been described under the Materials and Methods section. ${ }^{*}=\mathrm{P}<0.05,{ }^{* *}=\mathrm{P}<0.01$

fig4

A

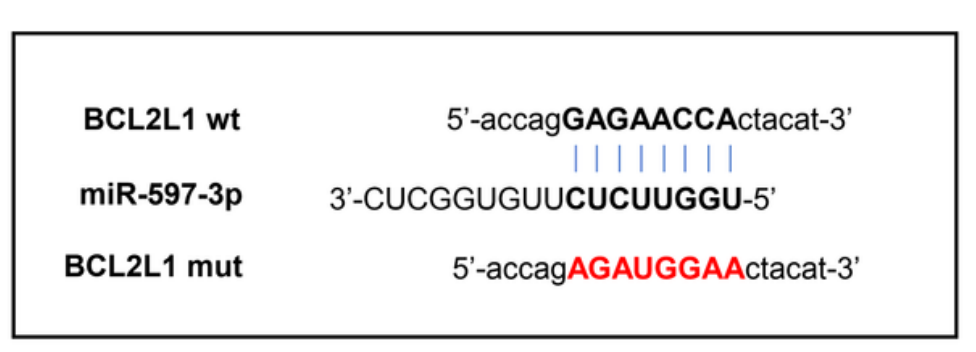

C

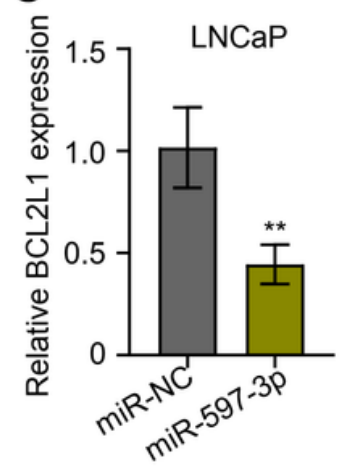

D

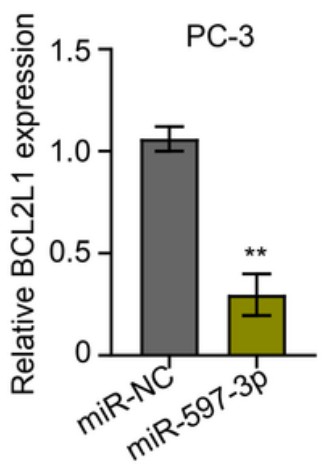

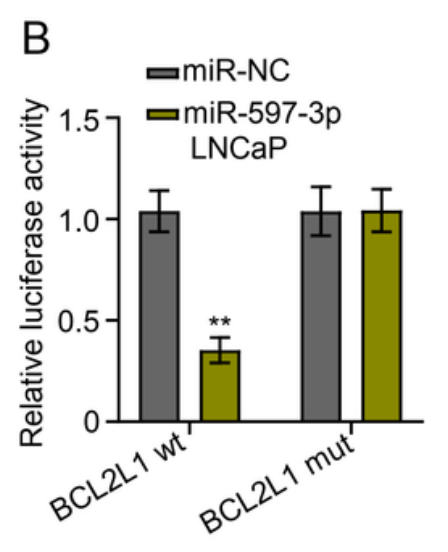

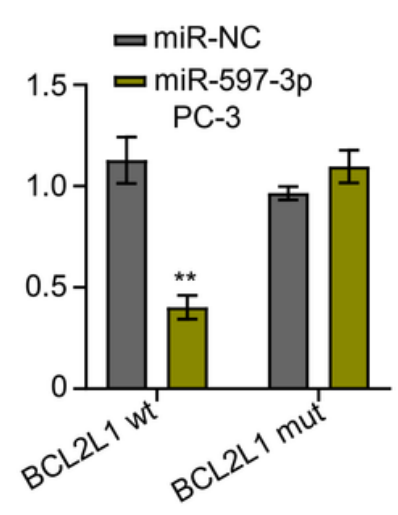

\section{E}

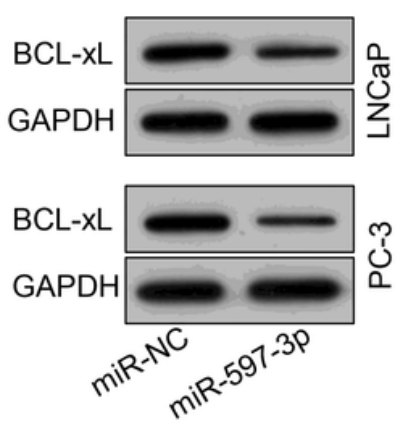

F
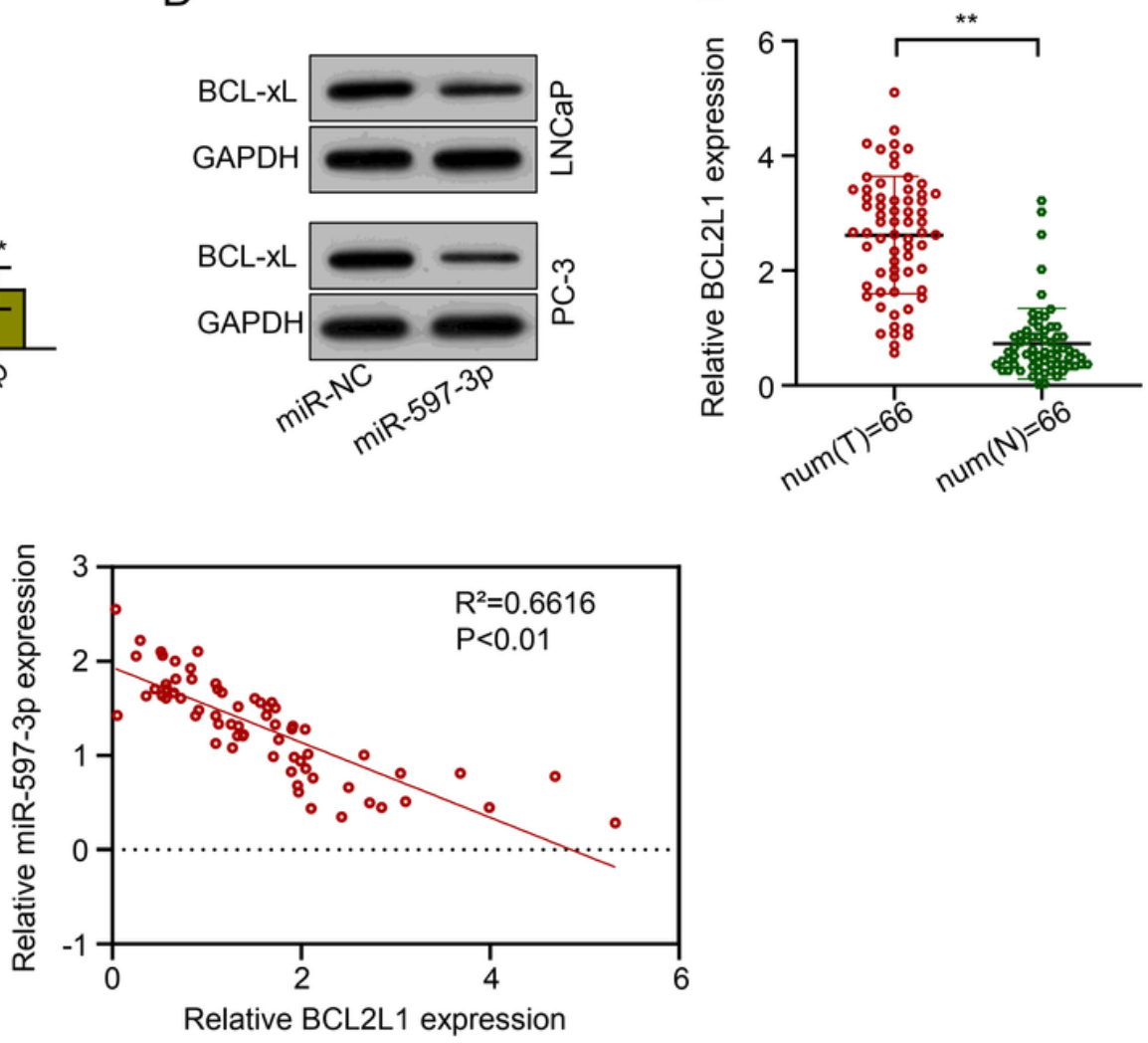

Figure 4 
miR-597-3p targeted BCL2L1. (A) Web based tool miRDB predicted that BCL2L1 3'UTR had binding site on miR-597-3P. (B) Luciferase experiment proved that BCL2L1 interacted with miR-597-3P. (C) Effect of miR-597-3P mimics on the mRNA expression of BCL2L1. (D) Effect of miR-597-3P mimics on the protein expression of BCL-xL. (E) The expression level of BCL2L1 in PRAD and adjacent tissue samples in 66 patients. (F) The expression of BCL2L1 was negatively correlated with miR-597-3p in PRAD clinical samples. Statistical analysis has been described under the Materials and Methods section. ${ }^{*}=P<0.05$, $\star *=\mathrm{P}<0.01$

fig5

A
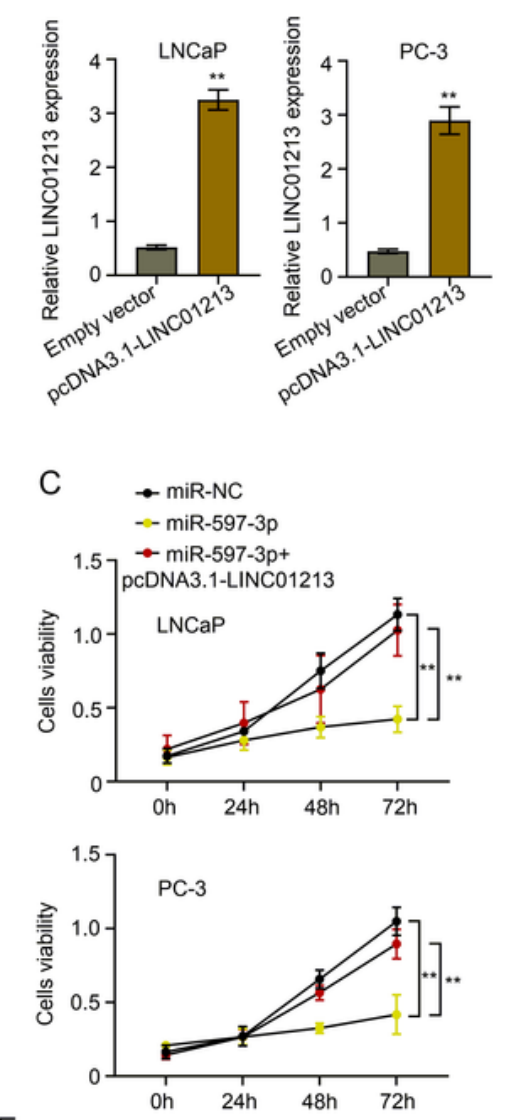

E
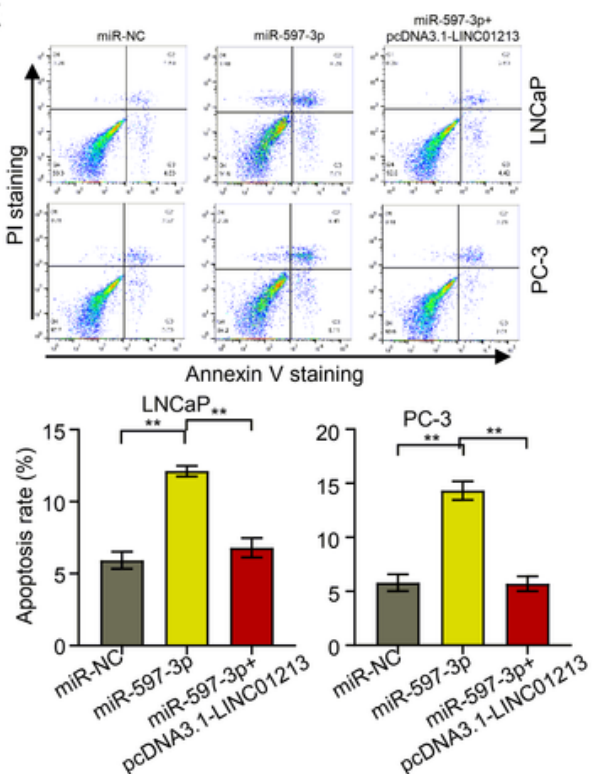

B

D
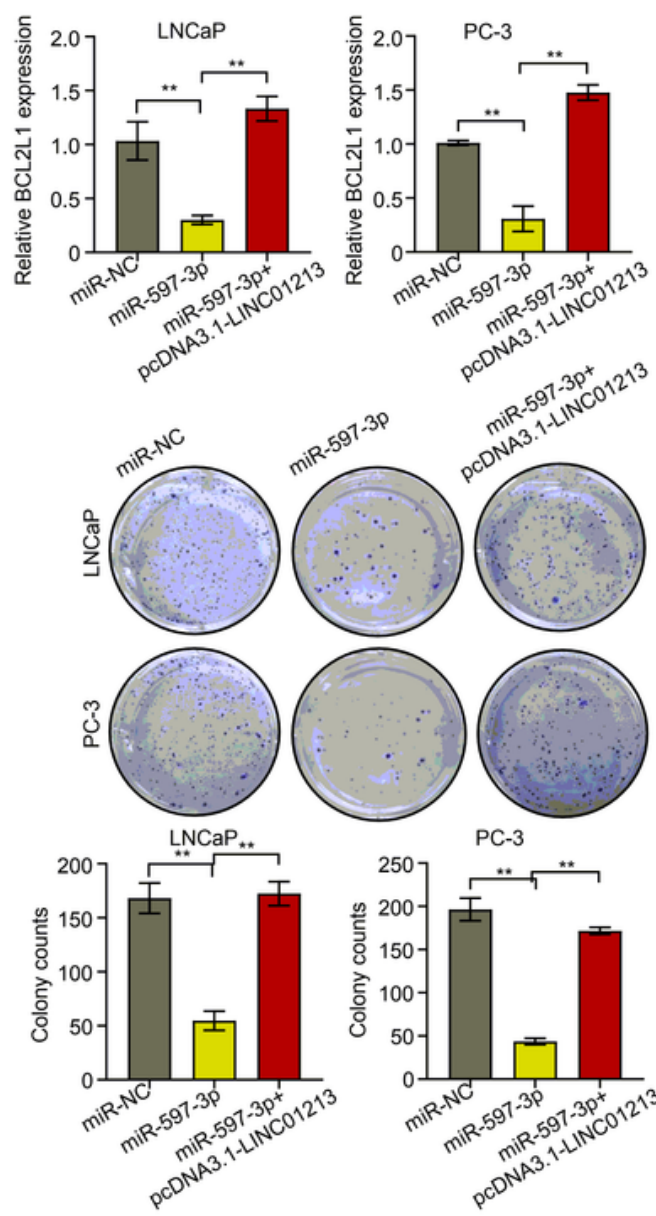

PC-3

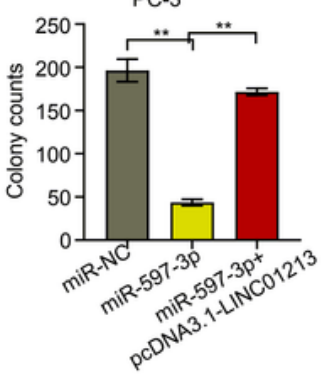

F

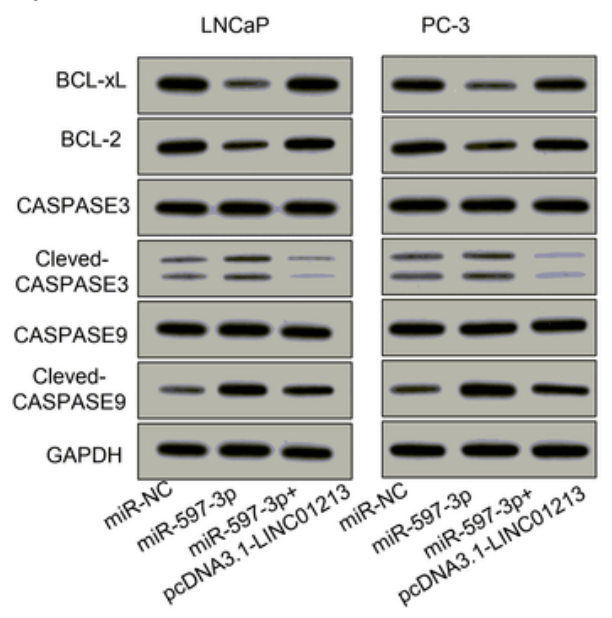




\section{Figure 5}

LINC01213 promotes PRAD through miR-597-3P/BCL2L1 axis. (A) Overexpression of LINC01213 in PRAD cell lines. (B) The expression level of BCL2L1 in different groups of LNCaP and PC-3 cells (miR-NC, miR597-3p mimics, miR-597-3p mimics+pcDNA3.1-LINC01213). CCK-8 (C) and colony formation assay (D) for the determination of survival and proliferation ability of PRAD cells after different treatments. (E) AnnexinV/PI cytometric assay for the determination of $\%$ apoptotic cells following different treatments. (F) Effect of different treatments on the protein expression of various mediators involved in cell survival and apoptosis in various groups (miR-NC, miR-597-3p mimics, miR-597-3p mimics+pcDNA3.1LINC01213). Statistical analysis has been described under the Materials and Methods section. ${ }^{*}=\mathrm{P}<$ $0.05, * *=\mathrm{P}<0.01$

\section{Supplementary Files}

This is a list of supplementary files associated with this preprint. Click to download.

- SupportingMaterialWB.pptx 